\title{
TRACING THE ORPHAN STREAM TO 55 kpc WITH RR LYRAE STARS
}

\author{
Branimir Sesar $^{1}$, Carl J. Grillmair ${ }^{2}$, Judith G. Cohen ${ }^{1}$, Eric C. Bellm ${ }^{1}$, Varun B. Bhalerao ${ }^{3}$, \\ David Levitan ${ }^{1}$, Russ R. Laher ${ }^{2}$, Eran O. OfeK ${ }^{4}$, Jason A. Surace ${ }^{2}$, Sumin Tang ${ }^{1}$, \\ Adam WaszczaK ${ }^{1}$, Shrinivas R. Kulkarni ${ }^{1}$, and Thomas A. Prince ${ }^{1}$ \\ ${ }^{1}$ Division of Physics, Mathematics and Astronomy, California Institute of Technology, Pasadena, CA 91125, USA; bsesar@astro.caltech.edu \\ ${ }^{2}$ Spitzer Science Center, California Institute of Technology, Pasadena, CA 91125, USA \\ ${ }^{3}$ Inter-University Centre for Astronomy and Astrophysics, Post Bag 4, Ganeshkhind, Pune 411 007, India \\ ${ }^{4}$ Benoziyo Center for Astrophysics, Weizmann Institute of Science, 76100 Rehovot, Israel \\ Received 2013 June 30; accepted 2013 August 8; published 2013 September 23
}

\begin{abstract}
We report positions, velocities, and metallicities of $50 a b$-type RR Lyrae (RRab) stars observed in the vicinity of the Orphan stellar stream. Using about 30 RRab stars classified as being likely members of the Orphan stream, we study the metallicity and the spatial extent of the stream. We find that RRab stars in the Orphan stream have a wide range of metallicities, from -1.5 dex to -2.7 dex. The average metallicity of the stream is -2.1 dex, identical to the value obtained by Newberg et al. using blue horizontal branch stars. We find that the most distant parts of the stream (40-50 kpc from the Sun) are about 0.3 dex more metal-poor than the closer parts (within $\sim 30 \mathrm{kpc}$ ), suggesting a possible metallicity gradient along the stream's length. We have extended the previous studies and have mapped the stream up to $55 \mathrm{kpc}$ from the Sun. Even after a careful search, we did not identify any more distant RRab stars that could plausibly be members of the Orphan stream. If confirmed with other tracers, this result would indicate a detection of the end of the leading arm of the stream. We have compared the distances of Orphan stream RRab stars with the best-fit orbits obtained by Newberg et al. We find that model 6 of Newberg et al. cannot explain the distances of the most remote Orphan stream RRab stars, and conclude that the best fit to distances of Orphan stream RRab stars and to the local circular velocity is provided by potentials where the total mass of the Galaxy within $60 \mathrm{kpc}$ is $M_{60} \sim 2.7 \times 10^{11} M_{\odot}$, or about $60 \%$ of the mass found by previous studies. More extensive modeling that would consider non-spherical potentials and the possibility of misalignment between the stream and the orbit is highly encouraged.
\end{abstract}

Key words: Galaxy: halo - Galaxy: kinematics and dynamics - Galaxy: structure - stars: variables: RR Lyrae

Online-only material: color figures, machine-readable tables

\section{INTRODUCTION}

One of the fundamental goals of Galactic astronomy is the determination of the Galaxy's gravitational potential because knowledge of it is required in any study of the dynamics or evolution of the Galaxy. An important tool in this undertaking is stellar tidal streams, which are remnants of accreted Milky Way satellites that were disrupted by tidal forces and stretched into filaments as they orbited in the Galaxy's potential. The orbits of stars in these streams are sensitive to the properties of the potential and thus allow us to constrain the potential over the range of distances spanned by the streams. Over the past several years, the GD-1 stream (Grillmair \& Dionatos 2006), the Sagittarius tidal streams (Majewski et al. 2003), and the Orphan stream (Grillmair 2006; Belokurov et al. 2007a) have been applied to this problem and have been used to constrain the circular velocity at the Sun's radius (Koposov et al. 2010), the total mass within $60 \mathrm{kpc}$ (Newberg et al. 2010), and the shape of the dark matter halo potential (Law \& Majewski 2010).

While each of the above streams is valuable in its own right, the Orphan stream seems to have several properties that make it almost ideal for inference of the Galactic potential. The stream is narrow $\left(\sim 2^{\circ}\right.$ wide), extends over $60^{\circ}$ of the sky, and probes distances from 20 to $50 \mathrm{kpc}$ from the Sun (Newberg et al. 2010). The misalignment between the stream and the orbit may be small enough that fitting an orbit directly to the stream has no significant consequences for the inferred shape of the Galactic potential (Sanders \& Binney 2013a, see also
Section 4). Its orbital plane is quite different from that of the Sagittarius streams, and thus the Orphan stream may be an important element in our attempts to understand the shape and orientation of the dark matter halo potential (the so-called halo conundrum; Law \& Majewski 2010). Because it spans distances from 20 to $50 \mathrm{kpc}$ from the Sun, the Orphan stream can be used to test new models of the Galactic potential that are based on the orbit of the Sagittarius stream (e.g., the model suggested by Vera-Ciro \& Helmi (2013) in which the shape of the dark matter halo potential transitions from oblate to triaxial at $\sim 30 \mathrm{kpc}$ ).

A state-of-the-art description of the Orphan stream and its orbit has been presented by Newberg et al. (2010). In their study, Newberg et al. used imaging data from the Sloan Digital Sky Survey (SDSS; York et al. 2000) and spectroscopic data from the Sloan Extension for Galactic Understanding and Exploration (SEGUE; Yanny et al. 2009b) to select main-sequence turnoff (MSTO) and blue horizontal branch (BHB) stars, and with them to trace the density, kinematics, and metallicity of the stream. Using these stars, they were able to trace the stream up to $46 \mathrm{kpc}$ from the Sun, after which the stream blended with the background and was no longer detectable in their data. In a more recent study, Casey et al. (2013) used low-resolution spectroscopy to identify several $\mathrm{K}$ giants, but these stars are associated with the closer part of the Orphan stream at $22 \mathrm{kpc}$ from the Sun.

It was quite unfortunate that the stream could not be traced to greater distances, as it prevented Newberg et al. (2010) from excluding certain models of the Galactic potential. As they stated 
in their Section 10.2, "if we could follow the Orphan stream just a little farther out into the halo, we would have a much better power to determine the halo mass, since the distances to the stream for each case diverge" (also see their Figures 12 and 14).

In this paper, we use $a b$-type RR Lyrae (RRab) stars to trace the Orphan stream in the northern Galactic hemisphere with a focus on tracing it beyond the $46 \mathrm{kpc}$ limit reached by Newberg et al. (2010). The main goal of this work is to support future studies of the Galactic potential by providing a clean sample of Orphan stream RR Lyrae stars with precise distances and radial velocities (RVs; better than $5 \%$ and $15 \mathrm{~km} \mathrm{~s}^{-1}$, respectively). The secondary goal of this work is to use the kinematics and positions of RR Lyrae stars associated with the Orphan stream to see whether new measurements exclude any of the models previously considered by Newberg et al. (2010).

Compared to MSTO, BHB, and K giant stars used in previous studies of the stream, RRab stars (fundamental-mode pulsators) have three important advantages. First, they are bright stars $\left(M_{r}=0.6 \mathrm{mag}\right.$ at $\left.[\mathrm{Fe} / \mathrm{H}]=-1.5 \mathrm{dex}\right)$ that can be detected at large distances $(5-120 \mathrm{kpc}$ for $14<r<21)$. Second, they are standard candles ( $\sim 5 \%$ uncertainty in distance; see Section 2.2), and third, they have distinct, saw-tooth-shaped light curves that make them easy to identify given multi-epoch observations (peak-to-peak amplitudes of $r \sim 1 \mathrm{mag}$ and periods of $\sim 0.6$ days). RR Lyrae type $c$ (RRc, first-overtone pulsators) stars have more sinusoidal light curves, which are less distinct and more difficult to separate from, for example, contact binary systems (e.g., see Figure 5 of Sesar et al. 2010). Furthermore, RRc stars are less numerous than RRab stars (the RRab to RRc ratio is about $3: 1)$. Due to these reasons, RRc stars are not used in this work.

Thanks to their distinct light curves, pure and highly complete samples ( $\gtrsim 95 \%$; Sesar et al. 2010) of RRab stars can be selected, allowing one to trace and efficiently ${ }^{5}$ follow up with spectroscopy even in quite diffuse and distant halo substructures (e.g., the Pisces Overdensity and the Cancer moving groups; Sesar et al. 2010, 2012). In contrast, samples of BHB stars selected using photometry only will be either incomplete or will have a non-negligible to severe contamination by non-BHB objects, ${ }^{6}$ resulting in unnecessary spectroscopic follow up of non-BHB objects. More relevant to the broader topic of tracing halo substructures, the contamination in samples of intrinsically sparse tracers (such as BHB or RR Lyrae stars), even as small as $25 \%$, can be problematic as it may cause the appearance of false halo substructures (for a detailed discussion of this problem, see Section 2.4 of Sesar et al. 2013).

The paper is organized as follows. In Section 2, we briefly describe the surveys and the catalogs from which the target RR Lyrae stars were selected, and then proceed to describe the spectroscopic data, their reduction, and the measurement of RVs and metallicities of observed RR Lyrae stars. In Section 3.1, we use metallicities and distances to identify likely members of Orphan stream. Likely members are then used to study the metallicity of the stream and its spatial extent in Sections 3.2 and 3.3. In Section 3.4, we compare the positions and velocities of likely Orphan stream members to best-fit orbits obtained by Newberg et al. (2010), and then present our conclusions in Section 4.

\footnotetext{
5 i.e., no observing time is wasted on non-RR Lyrae stars.

6 The best color-based selections of BHB stars achieve $\sim 75 \%$ purity at

$\sim 50 \%$ completeness (Sirko et al. 2004; Bell et al. 2010; Vickers et al. 2012).
}

\section{DATA}

\subsection{Overview of CSS, LINEAR, and PTF Surveys}

The RRab stars used in this paper come from three synoptic surveys: the Catalina Real-Time Sky Survey (CRTS), Lincoln Near Earth Asteroid Research (LINEAR) survey, and the Palomar Transient Factory (PTF). In the remainder of this section, we summarize each of these surveys, including an overview of the survey parameters and details on the quality of their photometry.

The CRTS $^{7}$ (Drake et al. 2009) uses three separate telescopes: the Catalina Sky Survey $0.7 \mathrm{~m}$ Schmidt (CSS), the Mount Lemmon Survey $1.5 \mathrm{~m}$ (MLS), and the Siding Spring Survey $0.5 \mathrm{~m}$ Schmidt (SSS). The fields of view are, respectively, $8.1 \mathrm{deg}^{2}, 1.2 \mathrm{deg}^{2}$, and $4.2 \mathrm{deg}^{2}$, with corresponding limiting magnitudes 19.5, 21.5, and 19.0 mag (see Table 1 of Larson 2007). The magnitude zero-point of CRTS photometry is tied to the Johnson $V$-band even though the observations are taken through a clear filter. ${ }^{8}$ The majority of data currently available is from CSS (Drake et al. 2013a), and has a typical cadence of one set of four exposures per night per field separated by $10 \mathrm{~min}$, repeated every two weeks. The uncertainty in CRTS photometry is $\sim 0.03 \mathrm{mag}$ for $V<16 \mathrm{mag}$ and rises to $0.1 \mathrm{mag}$ at $V \sim 19.2$ mag (see Figure 3 of Drake et al. 2013a).

The LINEAR survey ${ }^{9}$ (Stokes et al. 2000) uses two telescopes at the White Sands Missile Range for a synoptic survey primarily targeted at the discovery of near Earth objects. Each exposure covers $\sim 2 \mathrm{deg}^{2}$ to a $5 \sigma$ limiting magnitude of $r \sim 18$. Sesar et al. (2011b) re-calibrated the LINEAR data using the SDSS survey, resulting in 200 unfiltered observations per object (600 observations for objects within $\pm 10^{\circ}$ of the Ecliptic plane) for 25 million objects in the $9000 \mathrm{deg}^{2}$ of sky where the LINEAR and SDSS surveys overlap (roughly, the SDSS Galactic cap north of galactic latitude 30 and the SDSS Stripe 82 region). The photometric precision of LINEAR photometry is $0.03 \mathrm{mag}$ at the bright end $(r \sim 14)$ and $0.2 \mathrm{mag}$ at $r=18 \mathrm{mag}$.

The PTF ${ }^{10}$ (Law et al. 2009; Rau et al. 2009) is a synoptic survey designed to explore the transient sky. The project utilizes the 48 inch Samuel Oschin Schmidt Telescope on Mount Palomar. Each PTF image covers $7.26 \mathrm{deg}^{2}$ with a pixel scale of 1".01. The typical PTF cadence consists of two $60 \mathrm{~s}$ exposures separated by $\sim 1 \mathrm{hr}$ and repeated every one to five days. By 2013 June, PTF observed $\sim 11,000 \mathrm{deg}^{2}$ of sky at least 25 times in the Mould- $R$ filter ${ }^{11}$ (hereafter, the $R$-band filter), and about $2200 \mathrm{deg}^{2}$ in the SDSS $g^{\prime}$ filter. PTF photometry is calibrated to an accuracy of about 0.02 mag (Ofek et al. 2012a, 2012b) and light curves have relative precision of better than 10 mmag at the bright end, and about 0.2 mag at the survey limiting magnitude of $R=20.6$ mag. The relative photometry algorithm is described in Ofek et al. (2011, see their Appendix A).

To verify that the measurements taken by different surveys have the same magnitude zero-point, we have compared median PTF, LINEAR, and CSS magnitudes with SDSS $r$-band magnitudes. This comparison was done for non-variable sources with the SDSS $g-r$ color in the 0.2-0.3 mag range (average

\footnotetext{
7 http://crts.caltech.edu

8 The filter response of the CSS clear filter is closer to Johnson $R$-band than to Johnson $V$-band (A. Drake 2013, private communication).

9 Public access to LINEAR data is provided through the SkyDOT Web site (https://astroweb.lanl.gov/lineardb).

$10 \mathrm{http}: / / \mathrm{ptf} . c a l t e c h . e d u / i p t f$

11 The Mould- $R$ filter is similar in shape to the SDSS $r$-band filter, but shifted $27 \AA$ A redward.
} 


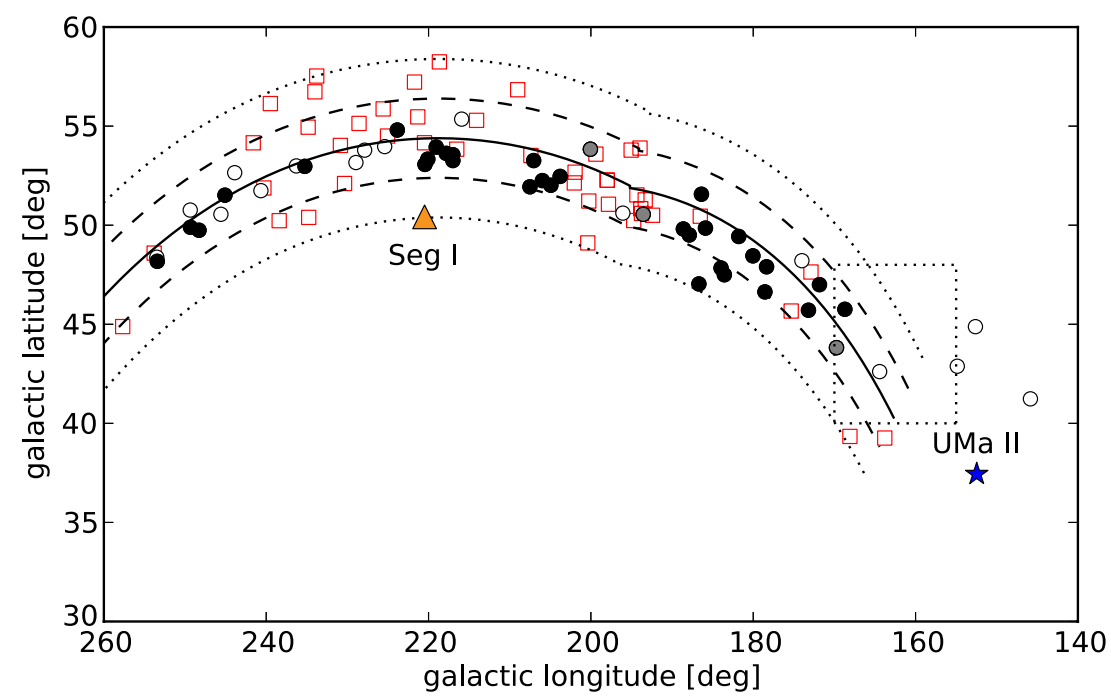

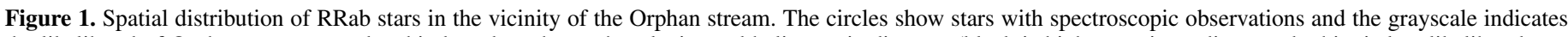

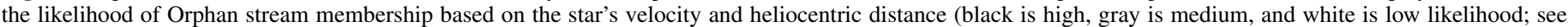

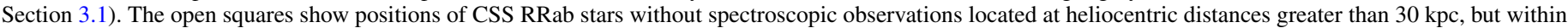

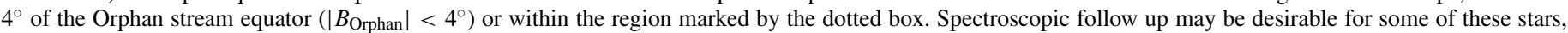

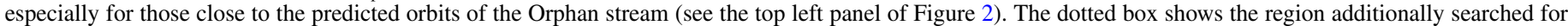

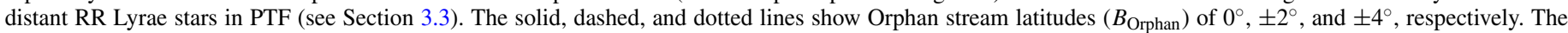

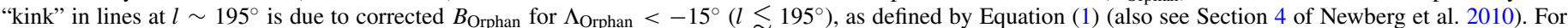

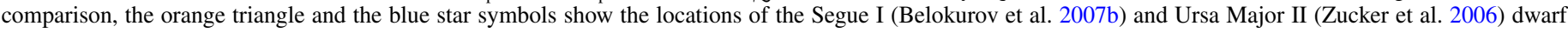
spheroidal (dSph) galaxies, respectively.

(A color version of this figure is available in the online journal.)

$g-r$ color of RR Lyrae stars). We found that the magnitudes of sources in this color range are offset by $\Delta=-0.05 \mathrm{mag}(\mathrm{PTF})$, $\Delta=0.05 \mathrm{mag}$ (LINEAR), and $\Delta=-0.05 \mathrm{mag}$ (CSS).

\subsection{Spectroscopic Targets}

In Figure 1, we show the spatial distribution of RRab stars targeted for spectroscopy. The targets were taken from CSS (Drake et al. 2013a), LINEAR (Sesar et al. 2013), and PTF catalogs of RR Lyrae stars (B. Sesar et al., in preparation), and are located in the vicinity of the Orphan stream.

Throughout this work we use the longitude $\Lambda_{\text {Orphan }}$ and latitude $B_{\text {Orphan }}$ defined in the coordinate system of the Orphan stream by Newberg et al. (2010, see their Section 2). As described in their Section 4 , for $\Lambda_{\text {Orphan }}<-15^{\circ}$ the Orphan latitude needs to be redefined as

$$
B_{\text {corr }}=B_{\text {Orphan }}+0.00628 \Lambda_{\text {Orphan }}^{2}+0.42 \Lambda_{\text {Orphan }}+5 .
$$

To allow reproduction of coordinates used in this work, we provide (as supplementary data) two functions written in Python that convert galactic to Orphan coordinates and vice versa.

Briefly, to select RR Lyrae stars from PTF we first searched for variable PTF sources that have SDSS colors consistent with colors of RR Lyrae stars (Equations (6)-(9) of Sesar et al. 2010). A period-finding algorithm was then applied to light curves of color-selected objects, and objects with periods in the range $0.2-0.9$ days were kept. Light curves were then phased (period-folded) and SDSS $r$-band RR Lyrae light curve templates constructed by Sesar et al. (2010) were fitted to phased data. Finally, we visually inspected template-fitted light curves to tag sources as RR Lyrae stars. A more detailed description of the selection of PTF RR Lyrae stars can be found in Section 3 of Sesar et al. (2012).

The positions and light curve parameters of RR Lyrae stars observed in this work are listed in Table 1. The heliocentric distances were calculated using the original flux-averaged magnitude, $\langle m\rangle_{\text {orig }}$, corrected for the ISM extinction $(r E x t)$ and the magnitude offset with respect to the SDSS $r$-band $(\Delta)$

$$
\langle m\rangle=\langle m\rangle_{\text {orig }}-r E x t-\Delta,
$$

where $r E x t$ is the extinction in the SDSS $r$-band calculated using the Schlegel et al. (1998) dust map, and $\Delta$ is $-0.05 \mathrm{mag}$ (PTF), $0.05 \mathrm{mag}$ (LINEAR), and $-0.05 \mathrm{mag}$ (CSS; see the last paragraph in Section 2.1).

The absolute magnitude of an RRab star, $M_{\mathrm{RR}}$ (Chaboyer 1999; Cacciari \& Clementini 2003), was calculated as

$$
M_{\mathrm{RR}}=(0.23 \pm 0.04)([\mathrm{Fe} / \mathrm{H}]+1.5)+(0.59 \pm 0.03),
$$

where $[\mathrm{Fe} / \mathrm{H}]$ is the spectroscopic metallicity of an RR Lyrae star (see Section 2.7). Following the discussion by Vivas \& Zinn (2006) (see their Section 4), we adopt $\sigma_{M_{V}}=$ $\sqrt{\left(\sigma_{M_{V}}^{[\mathrm{Fe} / \mathrm{H}]}\right)^{2}+\left(\Delta M_{V}^{\mathrm{ev}}\right)^{2}}=0.09 \mathrm{mag}$ as the uncertainty in absolute magnitude, where $\sigma_{M_{V}}^{[\mathrm{Fe} / \mathrm{H}]}=0.05$ mag is the uncertainty in Equation (3) due to uncertainty in measured spectroscopic metallicity $\left(\sigma_{[\mathrm{Fe} / \mathrm{H}]}=0.15 \mathrm{dex}\right.$, see Section 3.2), and $\Delta M_{V}^{\mathrm{ev}}=0.08$ mag is the uncertainty due to RR Lyrae evolution off the zero-age horizontal branch (Vivas \& Zinn 2006). Combined with a maximum of 0.05 mag uncertainty in the fluxaveraged magnitude $\langle m\rangle$, we find the uncertainty in the distance modulus to be $\sim 0.1 \mathrm{mag}$, or $\sim 5 \%$ in distance.

\subsection{Light Curve Parameters of CSS RR Lyrae Stars}

The light curve parameters of PTF and LINEAR RRab stars were simply adopted from papers describing their selection. However, the same could not be done for CSS RRab, as we describe below.

Following a visual inspection of phased light curves of CSS RRab stars (phased using periods and ephemeris values taken 
Table 1

Positions and Light Curve Parameters of RR Lyrae Targets

\begin{tabular}{|c|c|c|c|c|c|c|c|c|c|c|c|}
\hline Name & $\begin{array}{l}\text { R.A. }{ }^{a} \\
(\operatorname{deg})\end{array}$ & $\begin{array}{l}\text { Decl. }^{\mathrm{a}} \\
\text { (deg) }\end{array}$ & Survey & $\begin{array}{l}\text { Helio. Distance } \\
\qquad(\mathrm{kpc})\end{array}$ & $\begin{array}{c}\langle m\rangle^{\mathrm{b}} \\
(\mathrm{mag})\end{array}$ & $\begin{array}{l}\mathrm{rExt}^{\mathrm{c}} \\
(\mathrm{mag})\end{array}$ & $\begin{array}{l}\text { Amplitude } \\
\text { (mag) }\end{array}$ & $\begin{array}{c}m_{0}{ }^{\mathrm{d}} \\
(\mathrm{mag})\end{array}$ & $\begin{array}{l}\text { Period }^{\mathrm{e}} \\
\text { (days) }\end{array}$ & $\begin{array}{l}\mathrm{HJD}_{0}{ }^{\mathrm{f}} \\
\text { (days) }\end{array}$ & Member $^{\mathrm{g}}$ \\
\hline RR0 & 145.622246 & 66.493010 & LINEAR & 20.5 & 17.233 & 0.366 & 0.87 & 17.11 & 0.581007 & 53428.808929 & Low \\
\hline RR1 & 147.556760 & 60.410852 & LINEAR & 29.9 & 17.805 & 0.045 & 0.63 & 17.51 & 0.747907 & 54485.763320 & Low \\
\hline RR2 & 142.588413 & 59.743341 & LINEAR & 23.5 & 17.362 & 0.086 & 0.81 & 16.99 & 0.535057 & 52621.904999 & Low \\
\hline RR3 & 138.620393 & 53.065955 & LINEAR & 22.6 & 17.240 & 0.044 & 0.84 & 16.79 & 0.575657 & 54465.860645 & Low \\
\hline RR4 & 142.596437 & 49.440867 & CSS & 50.7 & 18.922 & 0.045 & 0.70 & 18.53 & 0.677648 & 54265.667221 & High \\
\hline RR6 & 143.840446 & 47.091109 & PTF & 54.9 & 19.081 & 0.032 & 1.02 & 18.49 & 0.530818 & 55887.972840 & High \\
\hline RR7 & 141.771831 & 46.359489 & PTF & 47.3 & 18.858 & 0.054 & 0.76 & 18.45 & 0.639017 & 55590.054047 & High \\
\hline RR8 & 145.212171 & 45.450505 & LINEAR & 20.2 & 17.129 & 0.053 & 0.81 & 16.71 & 0.654049 & 52620.938984 & Low \\
\hline RR9 & 144.271648 & 42.603354 & CSS & 41.0 & 18.517 & 0.038 & 0.77 & 18.11 & 0.567199 & 54913.653005 & High \\
\hline RR10 & 142.541300 & 42.570500 & CSS & 49.7 & 18.828 & 0.042 & 0.55 & 18.48 & 0.649151 & 54157.679811 & High \\
\hline
\end{tabular}

Notes. Tables 1 and 3 are available as a single table in the online version of the journal.

a Equatorial J2000.0 right ascension and declination from SDSS DR9 catalog.

b Flux-averaged magnitude (corrected for interstellar medium extinction and a magnitude offset with respect to SDSS $r$-band, as $\langle m\rangle=$ $\langle m\rangle_{\text {not corrected }}-\mathrm{rExt}-\Delta$, see Section 2.2).

${ }^{c}$ Extinction in the SDSS $r$-band calculated using the Schlegel et al. (1998) dust map.

d Magnitude at the epoch of maximum brightness.

e Period of pulsation.

${ }^{\mathrm{f}}$ Reduced Heliocentric Julian Date of maximum brightness (HJD - 2400000).

g Probability of being a member of the Orphan stream.

(This table is available in its entirety in a machine-readable form in the online journal. A portion is shown here for guidance regarding its form and content.)

from Table 1 of Drake et al. 2013a), we noticed that the phased light curves did not peak at phase of zero, as is the convention for RR Lyrae stars, but at phases that varied from star to star. This prevents accurate determination of the phase of pulsation, which is important when subtracting the velocity due to pulsations from the measured RV (see Section 2.6). We traced this problem to incorrect ephemeris values listed in Table 1 of Drake et al. 2013a, and notified Drake et al. of this issue.

To determine the correct epoch of maximum light for CSS RRab stars, we fitted the SDSS $r$-band RRab light curve templates of Sesar et al. (2010) to phased CSS light curves. The light curve parameters obtained using this procedure, such as the epoch of maximum light (needed for accurate estimation of the phase) and the flux-averaged magnitude (needed for distance estimates), are listed in Table 1 and are used hereafter instead of the ones provided by Drake et al. (2013a). For comparison, the newly derived light curve amplitudes and the ones listed in Table 1 of Drake et al. (2013a) agree to 3\%.

To support future spectroscopic follow up of RRab stars in the vicinity of the Orphan stream, the positions and light curve parameters of CSS RRab stars without spectroscopic observations (open squares in Figures 1 and 2) are provided in Table 2.

\subsection{Spectroscopic Observations}

The spectroscopic observations were obtained using the blue channel of the Double Spectrograph (DBSP; Oke \& Gunn 1982) mounted on the Palomar $5.1 \mathrm{~m}$ telescope. A 600 lines $\mathrm{mm}^{-1}$ grating and a $5600 \AA$ dichroic were used, providing a resolution of $R=1360$ and a spectral range from $3800 \AA$ to $5700 \AA$.

Target RR Lyrae stars were observed over the course of several nights in $600 \mathrm{~s}$ to $900 \mathrm{~s}$ exposures. In order to avoid the discontinuity in the RV curve near maximum light, the observations were scheduled to target stars between phases of 0.1 and 0.85 of their pulsation cycle, with earlier phases being preferred as the stars are brighter then.
In addition, we also targeted several equivalent-width (EW) standard stars (see Table 6 of Layden 1994), as well as a few bright RR Lyrae stars with well-determined spectroscopic metallicities. The EW standard stars were observed in order to transform EWs measured from DBSP spectra to Layden's (1994) system (see his Section 3.4.3 and our Section 2.7 below). The bright RR Lyrae stars (VY Ser, RR Lyr, ST Boo, and VX Her) were observed in order to validate the metallicity measurements described in Section 2.7.

\subsection{Data Reduction and Calibration}

All data were reduced with standard IRAF $^{12}$ routines, and spectra were extracted using an optimal (inverse varianceweighted) method (Horne 1986). The wavelength calibration of spectra was done using a set of FeAr arc lines, and the spectra were flux-calibrated. The signal-to-noise ratio $(\mathrm{S} / \mathrm{N})$ of the spectra ranged from 10 to 30 at $4750 \AA$.

To correct for the possible wavelength shift in spectra during an exposure (e.g., due to instrument flexure), we measured the wavelengths $\left(\lambda_{\text {obs }}\right)$ of three $[\mathrm{Hg} \mathrm{I}]$ sky lines and one [O I] sky line, and then adjusted the zero-point of each spectrum by the mean of $\Delta \lambda=\lambda_{\text {obs }}-\lambda_{\text {lab }}$, where $\lambda_{\text {lab }}=4046.565,4358.335$, 5460.750 , and $5577.340 \AA$ are the laboratory wavelengths of $[\mathrm{Hg} \mathrm{I}]$ and $\left[\mathrm{O}_{\mathrm{I}}\right]$ sky lines when observed in air. The uncertainty in the zero-point of wavelength calibration was estimated as the standard error of the mean of $\Delta \lambda$ (i.e., standard deviation of $\Delta \lambda$ divided by two). On average, this uncertainty is $\sim 0.08 \AA$, or $\sim 5 \mathrm{~km} \mathrm{~s}^{-1}$ at $4750 \AA$.

\subsection{Center-of-mass Velocities}

The center-of-mass velocity (hereafter, the systemic velocity $v_{\text {helio }}$ ), is the line-of-sight velocity of an RR Lyrae star that one

12 IRAF is distributed by the National Optical Astronomy Observatory, which is operated by the Association of Universities for Research in Astronomy (AURA), Inc., under cooperative agreement with the National Science Foundation (http://iraf.noao.edu). 

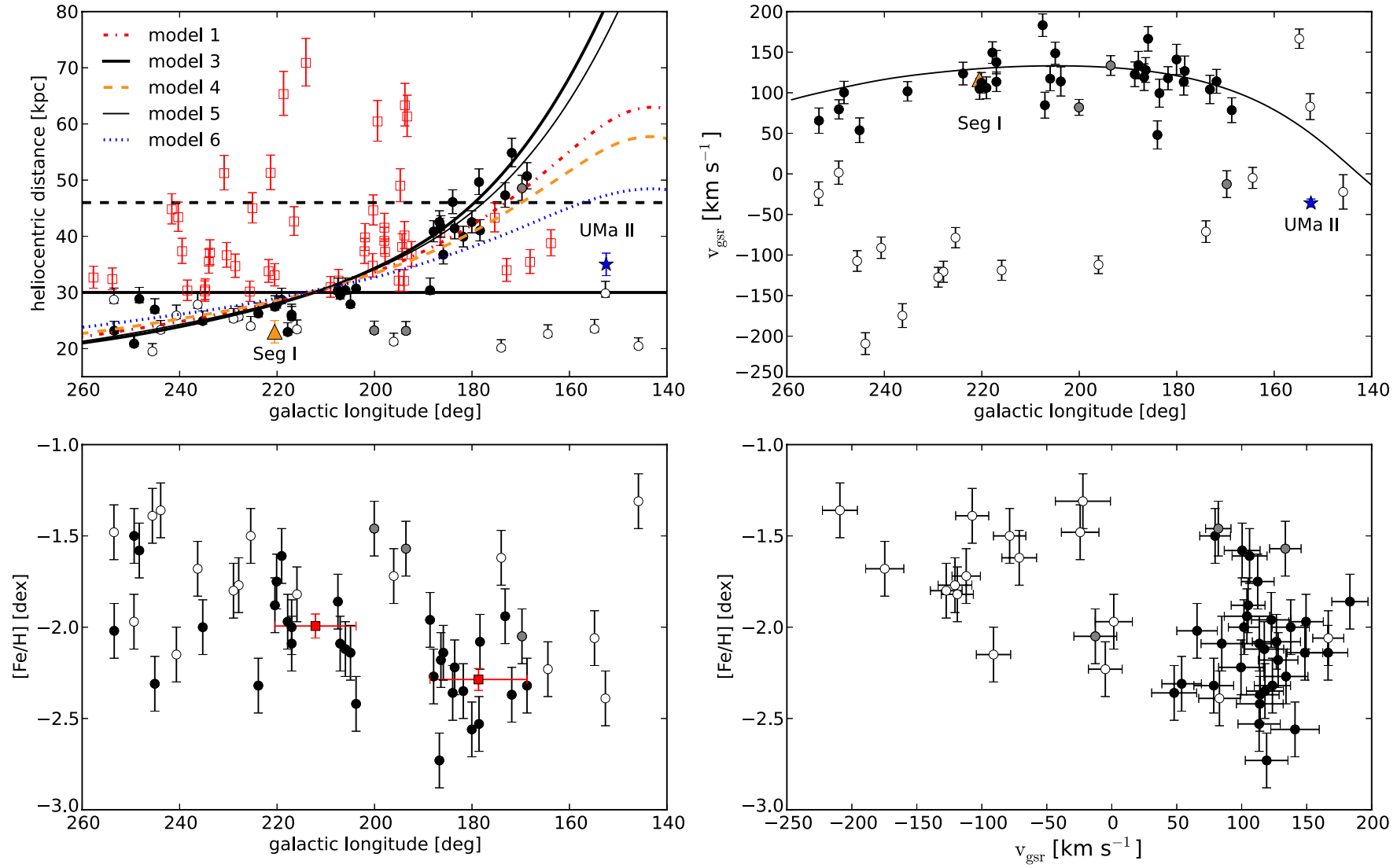

Figure 2. Top left: heliocentric distances vs. galactic longitude for RRab stars in the vicinity of the Orphan stream. The meaning of symbols is the same as in Figure 1. The error bars reflect the uncertainty of $0.1 \mathrm{mag}$ in distance modulus, or $\sim 5 \%$ in distance. The heliocentric distances for CSS RRab stars without spectroscopic observations (open squares) were calculated assuming $[\mathrm{Fe} / \mathrm{H}]=-2.0$ dex (uncertainty in distance is $\sim 7 \%$ ). The heliocentric distances of Segue I and Ursa Major II were taken from Dall'Ora et al. (2012) and Belokurov et al. (2007b), respectively. The lines show some of the best-fit orbits of the Orphan stream obtained by Newberg et al. (2010), assuming different models for the potential (see Table 4 and Section 3.4 for a description of their models; the lines for models 2 and 7 are not shown due to their similarity to the lines for models 3 and 5, respectively). The solid horizontal line at $30 \mathrm{kpc}$ shows the distance beyond which the number density of stars associated with the "smooth" stellar halo rapidly decreases (Sesar et al. 2011a), and the dashed horizontal line at 46 kpc shows the maximum distance probed by Newberg et al. (2010). Top right: Galactic standard of rest velocities vs. galactic longitude. The line shows the best-fit orbit of Newberg et al. (2010) assuming a potential defined by their model 3. For clarity, other orbits of Newberg et al. (2010) are not plotted because they are indistinguishable from the plotted one given the range of galactic longitudes probed here and given the uncertainties in velocities of RR Lyrae stars. The majority of stars associated with the Orphan stream have $v_{\mathrm{gsr}} \sim 100 \mathrm{~km} \mathrm{~s}^{-1}$. The stars with $v_{\mathrm{gsr}} \sim-120 \mathrm{~km} \mathrm{~s}^{-1}$ are likely associated with the Sagittarius tidal stream. The velocities of Segue I (orange triangle at $l \sim 220^{\circ}$ ) and Ursa Major II (blue star symbol) were taken from Martin et al. (2007) and Martinez et al. (2011), respectively. Bottom left: metallicity vs. galactic longitude. The error bars are set to 0.15 dex. The squares show the mean metallicity for two groups of RR Lyrae stars with high likelihoods of being members of the Orphan stream. The vertical error bars show the standard error of the mean and the horizontal error bars indicate the width of bins. The difference between the two mean metallicities is $0.3 \mathrm{dex}$, suggesting a possible metallicity gradient (the standard error of the mean is $\sim 0.06 \mathrm{dex}$ ). Bottom right: metallicity vs. the velocity in the Galactic standard of rest. Note how the velocity is a better criterion for separating Orphan and non-Orphan RR Lyrae stars than the metallicity. RR Lyrae stars associated with the Orphan stream are on average metal-poor (mean $[\mathrm{Fe} / \mathrm{H}]=-2.1 \mathrm{dex}$ ), and have metallicities from -1.5 dex to -2.7 dex (standard deviation is 0.3 dex). The group of stars with $v_{\text {gsr }} \sim-120 \mathrm{~km} \mathrm{~s}^{-1}$ and $[\mathrm{Fe} / \mathrm{H}]=-1.8$ dex has the velocity and the metallicity consistent with that of the Sagittarius leading tidal stream (Yanny et al. 2009a). (A color version of this figure is available in the online journal.)

Table 2

Positions and Light Curve Parameters of CSS RRab Stars without Spectroscopic Observations

\begin{tabular}{lcccccccc}
\hline \hline $\begin{array}{l}\text { R.A. }^{\mathrm{a}} \\
(\mathrm{deg})\end{array}$ & $\begin{array}{c}\text { Decl. }^{\mathrm{a}} \\
(\mathrm{deg})\end{array}$ & $\begin{array}{c}\text { Helio. Distance } \\
(\mathrm{kpc})\end{array}$ & $\begin{array}{c}\langle m\rangle^{\mathrm{c}} \\
(\mathrm{mag})\end{array}$ & $\begin{array}{c}\mathrm{rExt}^{\mathrm{d}} \\
(\mathrm{mag})\end{array}$ & $\begin{array}{c}\text { Amplitude } \\
(\mathrm{mag})\end{array}$ & $\begin{array}{c}m_{0}{ }^{\mathrm{e}} \\
(\mathrm{mag})\end{array}$ & $\begin{array}{c}\text { Period }^{\mathrm{f}} \\
(\text { days })\end{array}$ & $\begin{array}{c}\mathrm{HJD}_{0}{ }^{\mathrm{g}} \\
(\mathrm{days})\end{array}$ \\
\hline 133.171530 & 54.068280 & 38.8 & 18.412 & 0.059 & 0.67 & 18.05 & 0.596552 & 53745.681875 \\
132.773220 & 50.755860 & 35.4 & 18.217 & 0.069 & 0.95 & 17.69 & 0.652085 & 54946.735650 \\
\hline
\end{tabular}

Notes.

${ }^{\text {a }}$ Equatorial J2000.0 right ascension and declination.

${ }^{\mathrm{b}}$ Calculated assuming $[\mathrm{Fe} / \mathrm{H}]=-2.0 \mathrm{dex}$ and $M_{\mathrm{RR}}=0.47 \mathrm{mag}$.

${ }^{\mathrm{c}}$ Flux-averaged magnitude (corrected for interstellar medium extinction and a magnitude offset with respect to SDSS $r$-band, as $\langle m\rangle=$ $\langle m\rangle_{\text {not corrected }}-\mathrm{rExt}-\Delta$, see Section 2.2).

${ }^{d}$ Extinction in the SDSS $r$-band calculated using the Schlegel et al. (1998) dust map.

e Magnitude at the epoch of maximum brightness.

${ }^{\mathrm{f}}$ Period of pulsation.

g Reduced Heliocentric Julian Date of maximum brightness (HJD - 2400000).

(This table is available in its entirety in a machine-readable form in the online journal. A portion is shown here for guidance regarding its form and content.) 
would measure if its atmosphere was at rest. As described below, we first measure the heliocentric RVs of $\mathrm{H} \gamma$ and $\mathrm{H} \beta$ Balmer lines $\left(v_{\mathrm{H} \gamma}\right.$ and $\left.v_{\mathrm{H} \beta}\right)$, and then fit them to template $\mathrm{RV}$ curves as a function of phase to obtain the systemic velocity. We use Balmer lines because they are least affected by blending in low resolution DBSP spectra. The velocities are measured separately for each Balmer line because the lines form at different heights in the atmosphere and have different velocities as a function of pulsation phase (Oke et al. 1962; Sesar 2012).

The RVs were measured by cross-correlating observed spectra with about 350 synthetic spectra (hereafter, template spectra) selected from the Munari et al. (2005) spectral library. The template spectra have a linear dispersion of $1 \AA$ A per pixel and encompass the range of metallicities, surface gravities, and effective temperatures covered by RRab stars $\left(5000<T_{\text {eff }} / \mathrm{K}<10,000\right.$, $1.5<\log g<3.5,-2.5<[\mathrm{Fe} / \mathrm{H}] / \mathrm{dex}<-0.5)$.

The cross-correlation was done separately for two spectral regions, one centered on $\mathrm{H} \gamma(4160-4630 \AA)$ and the other centered on $\mathrm{H} \beta$ (4630-5000 $\mathrm{A}$ ), using an IDL program written by V. Bhalerao (getvel ${ }^{13}$; Bhalerao 2012). Briefly, this program takes a template spectrum, resamples it to the dispersion of the observed spectrum, convolves the resampled spectrum with an appropriate Line Spread Function, and finds the velocity shift for which the $\chi^{2}$ per degree of freedom between the template and the observed spectrum is the lowest. The velocity obtained from the template with the lowest $\chi^{2}$ per degree of freedom is adopted as the best-fit $\mathrm{RV}\left(v_{\mathrm{H} \gamma}\right.$ or $\left.v_{\mathrm{H} \beta}\right)$. The uncertainty in the best-fit RV, $\sigma_{c c}$, was estimated as the velocity range around the best-fit RV within which the $\chi^{2}$ per degree of freedom increases by 1 . On average, this uncertainty was about $13 \mathrm{~km} \mathrm{~s}^{-1}$.

The systemic velocities were determined by fitting the template RV curves to measured $\mathrm{H} \gamma$ and $\mathrm{H} \beta$ velocities. For this purpose, we used template $\mathrm{H} \gamma$ and $\mathrm{H} \beta$ velocity curves constructed by Sesar (2012). Given an RRab star with a Johnson $V$-band light curve amplitude of $A_{V}$, the amplitude of a Balmer line RV template $\left(A_{\mathrm{rv}}\right)$ was set using the relations of Sesar (2012)

$$
\begin{aligned}
& A_{\mathrm{rv}}^{\mathrm{H} \gamma}=46.1( \pm 2.5) A_{V}+38.5( \pm 2.4), \sigma_{\mathrm{fit}}=2.8 \mathrm{~km} \mathrm{~s}^{-1} \\
& A_{\mathrm{rv}}^{\mathrm{H} \beta}=42.1( \pm 2.5) A_{V}+51.1( \pm 2.4), \sigma_{\mathrm{fit}}=3.0 \mathrm{~km} \mathrm{~s}^{-1}
\end{aligned}
$$

The scaled template was then shifted in velocity to match the $v_{\mathrm{H} \gamma}$ or $v_{\mathrm{H} \beta}$ measurement at the corresponding phase; the systemic velocity is that of the shifted template at phase 0.5 . The $V$-band light curve amplitudes of observed RRab stars were calculated from their $R$-band light curve amplitudes as $A_{V}=1.21 A_{R}$ (see Section 5 of Sesar 2012).

For each star, the above procedure returns two estimates of the systemic velocity, one based on $\mathrm{H} \gamma\left(v_{\text {helio, } \mathrm{H} \gamma}\right)$ and the other one based on $\mathrm{H} \beta \mathrm{RV}\left(v_{\text {helio, } \mathrm{H} \beta}\right)$. Following Sesar (2012), the variance of a systemic velocity was calculated as

$$
\begin{aligned}
\sigma_{v}^{2} & =\sigma_{c c}^{2}+\sigma_{\text {model }}^{2} \\
& =\sigma_{c c}^{2}+\left(A_{\mathrm{rv}}+\sigma_{\mathrm{fit}}\right)^{2}\left[\sigma_{\text {template }}^{2}\left(\Phi_{\mathrm{obs}}\right)+(0.1 k)^{2}\right],
\end{aligned}
$$

where $k=1.54$ and $k=1.42$ for $\mathrm{H} \beta$ and $\mathrm{H} \gamma$ RV templates, respectively. The $\sigma_{\text {template }}^{2}\left(\Phi_{\text {obs }}\right)$ term is the variance of the template at the phase of observation (see bottom plots in Figure 1 of Sesar 2012), and $\sigma_{c c}^{2}$ is the variance of the best-fit heliocentric $\mathrm{RV}\left(v_{\mathrm{H} \gamma}\right.$ or $\left.v_{\mathrm{H} \beta}\right)$. On average, $\sigma_{\text {model }}$ is about $13 \mathrm{~km} \mathrm{~s}^{-1}$.

\footnotetext{
13 http://www.iucaa.ernet.in/ varunb/getvel/
}

The final systemic velocity of an RRab star was obtained by averaging the estimates based on two Balmer lines, where each estimate was weighted by the inverse of its variance

$$
v_{\text {helio }}=\frac{v_{\text {helio }, \mathrm{H} \gamma} / \sigma_{v, \mathrm{H} \gamma}^{2}+v_{\text {helio, } \mathrm{H} \beta} / \sigma_{v, \mathrm{H} \beta}^{2}}{1 / \sigma_{v, \mathrm{H} \gamma}^{2}+1 / \sigma_{v, \mathrm{H} \beta}^{2}} .
$$

The uncertainty in the final systemic velocity was calculated by adding (in quadrature) the uncertainty in the zero-point of wavelength calibration $\left(\sigma_{\mathrm{zpt}}\right)$ and the uncertainty in the weighted mean (i.e., $v_{\text {helio }}$ )

$$
\sigma_{\text {helio }}=\sqrt{\sigma_{\mathrm{zpt}}^{2}+\frac{1}{1 / \sigma_{v, \mathrm{H} \gamma}^{2}+1 / \sigma_{v, \mathrm{H} \beta}^{2}}} .
$$

The final systemic velocities and their uncertainties are listed in Table 3. In addition to line-of-sight velocities, Table 3 also lists proper motions taken from the Munn et al. (2004) catalog.

\subsection{Spectroscopic Metallicities}

Spectroscopic metallicities were measured following the method and calibration of Layden (1994) which involves comparing the pseudo-equivalent width of [CaII] $\mathrm{K}$ line, $W(\mathrm{~K})$, against the mean pseudo-equivalent widths of $\beta, \gamma$, and $\delta$ Balmer lines, $W(\mathrm{H})$.

The pseudo-equivalent widths (hereafter, EWs) of the [Ca II] $\mathrm{K}$ line and Balmer lines were measured from DBSP spectra (normalized to the pseudo-continuum) using the EWIMH program ${ }^{14}$ written by A. Layden. Measured EWs $\left(W^{\prime}(\mathrm{K}), W^{\prime}(\mathrm{H} \delta), W^{\prime}(\mathrm{H} \gamma)\right.$, and $W^{\prime}(\mathrm{H} \beta)$ ) were then transformed to Layden's (1994) EW system using the following relations:

$$
\begin{gathered}
W(\mathrm{~K})=1.11 W^{\prime}(\mathrm{K})-0.24 \\
W(\mathrm{H} \delta)=0.87 W^{\prime}(\mathrm{H} \delta)+1.02 \\
W(\mathrm{H} \gamma)=1.26 W^{\prime}(\mathrm{H} \gamma)-1.00 \\
W(\mathrm{H} \beta)=1.02 W^{\prime}(\mathrm{H} \beta)+1.22 .
\end{gathered}
$$

The above equations were derived by comparing EWs of eight EW standard stars observed with DBSP, with the EW values measured by Layden (1994) and listed in his Table 6. After the transformation, $W(\mathrm{~K})$ was corrected for interstellar [Ca II] absorption using the Beers (1990) model

$$
W\left(\mathrm{~K}_{0}\right)=W(\mathrm{~K})-W_{\max }\left(1-e^{-|z| / h}\right) / \sin |b|,
$$

where $W_{\max }=0.192 \AA, h=1.081 \mathrm{kpc}, b$ is the Galactic latitude, and $z$ is the height above the Galactic plane in kpc.

The spectroscopic metallicity was calculated as

$$
[\mathrm{Fe} / \mathrm{H}]=\frac{W\left(\mathrm{~K}_{0}\right)-a-b W(\mathrm{H})}{c+d W(\mathrm{H})},
$$

where $a=13.858, b=-1.185, c=4.228$, and $d=-0.32$ (see Table 8 in Layden 1994). Equation (14) was obtained

\footnotetext{
${ }^{14}$ http://physics.bgsu.edu/ layden/ASTRO/DATA/EXPORT/EWIMH/ ewimh.htm
} 
Table 3

Line-of-sight Velocities, Proper Motions, and Metallicities

\begin{tabular}{|c|c|c|c|c|c|c|c|c|c|c|}
\hline Name & $\begin{array}{l}\text { R.A. }^{a} \\
(\operatorname{deg})\end{array}$ & $\begin{array}{l}\text { Decl. }^{\mathrm{a}} \\
\text { (deg) }\end{array}$ & $\begin{array}{l}\text { Helio. Distance } \\
(\mathrm{kpc})\end{array}$ & $\begin{array}{c}v_{\text {helio }}{ }^{\mathrm{a}} \\
\left(\mathrm{km} \mathrm{s}^{-1}\right)\end{array}$ & $\begin{array}{c}v_{\mathrm{gsr}}^{\mathrm{b}} \\
\left(\mathrm{km} \mathrm{s}^{-1}\right)\end{array}$ & $\begin{array}{c}\mu_{l}^{\mathrm{c}} \\
\left(\operatorname{mas~yr}^{-1}\right)\end{array}$ & $\begin{array}{c}\mu_{b}{ }^{\mathrm{d}} \\
\left(\text { mas yr}^{-1}\right)\end{array}$ & $\begin{array}{c}\mu_{\mathrm{err}}{ }^{\mathrm{e}} \\
\left(\operatorname{mas~yr}^{-1}\right)\end{array}$ & $\begin{array}{l}{[\mathrm{Fe} / \mathrm{H}]} \\
(\mathrm{dex})\end{array}$ & Member $^{f}$ \\
\hline RR0 & 145.622246 & 66.493010 & 20.5 & $-114.9 \pm 21.2$ & -22.2 & 4.82 & 1.81 & 2.68 & -1.31 & Low \\
\hline RR1 & 147.556760 & 60.410852 & 29.9 & $11.5 \pm 15.9$ & 82.9 & 9.77 & 0.78 & 3.12 & -2.39 & Low \\
\hline RR2 & 142.588413 & 59.743341 & 23.5 & $99.0 \pm 11.9$ & 166.6 & 4.22 & 0.16 & 2.84 & -2.06 & Low \\
\hline RR3 & 138.620393 & 53.065955 & 22.6 & $-46.6 \pm 13.0$ & -5.0 & 1.36 & 1.53 & 2.81 & -2.23 & Low \\
\hline RR4 & 142.596437 & 49.440867 & 50.7 & $50.0 \pm 15.3$ & 78.5 & 1.03 & 2.77 & 3.35 & -2.32 & High \\
\hline RR5 & 139.486634 & 49.043981 & 48.6 & $-38.9 \pm 16.5$ & -12.7 & 6.64 & -1.07 & 3.57 & -2.05 & Medium \\
\hline RR6 & 143.840446 & 47.091109 & 54.9 & $94.3 \pm 14.7$ & 114.1 & -3.49 & -4.27 & 3.35 & -2.37 & High \\
\hline RR7 & 141.771831 & 46.359489 & 47.3 & $87.7 \pm 17.4$ & 104.0 & 2.54 & -0.72 & 3.43 & -1.94 & High \\
\hline RR8 & 145.212171 & 45.450505 & 20.2 & $-85.1 \pm 13.3$ & -71.2 & 2.07 & 0.98 & 2.68 & -1.62 & Low \\
\hline RR9 & 144.271648 & 42.603354 & 41.0 & $124.2 \pm 18.5$ & 126.7 & -3.10 & -1.34 & 3.37 & -2.08 & High \\
\hline RR10 & 142.541300 & 42.570500 & 49.7 & $111.6 \pm 16.3$ & 113.4 & -0.06 & -5.99 & 3.50 & -2.53 & High \\
\hline
\end{tabular}

Notes. Tables 1 and 3 are available as a single table in the online version of the journal.

${ }^{a}$ Heliocentric systemic velocity and its uncertainty.

b Velocity in the Galactic standard of rest (see Section 3)

${ }^{c}$ Proper motion in direction of galactic longitude.

d Proper motion in direction of galactic latitude.

e Uncertainty in each proper motion component.

${ }^{\mathrm{f}}$ Probability of being a member of the Orphan stream.

(This table is available in its entirety in a machine-readable form in the online journal. A portion is shown here for guidance regarding its form and content.)

by inverting Equation (7) of Layden (1994). The metallicities measured using this method are listed in Table 3.

To validate the measured metallicities, we observed four bright RR Lyrae stars (VY Ser, RR Lyr, ST Boo, and VX Her) that have well-determined spectroscopic metallicities $(-1.9<$ $[\mathrm{Fe} / \mathrm{H}] /$ dex $<-1.3)$. An unweighted linear least-squares fit between metallicities measured by us and by Layden (1994) returned a slope consistent with one (1.05), and an intercept consistent with zero (0.01). The root-mean-square (rms) scatter of the fit was $0.07 \mathrm{dex}$, which we adopt as the systematic uncertainty of this method. Thus, we conclude that our metallicities are on the Layden (1994) metallicity system, which is tied to the Zinn \& West (1984) globular cluster abundance scale.

\subsection{Comparison with SDSS Measurements}

SDSS and SEGUE spectra exist for 27 RRab stars from our spectroscopic sample. For these spectra, the SEGUE Stellar Parameters Pipeline (SSPP; Lee et al. 2008, 2011) provides metallicities and RVs. Before we compare our measurements to the ones supplied by SSPP, there are three important points that need to be made.

First, SDSS spectra from which SSPP measurements are derived consist of multiple exposures, usually taken back-toback but sometimes even spread over days (Bickerton et al. 2012) (i.e., obtained at vastly different pulsation phases). Since the exposures were taken without the knowledge of the pulsation phase, some of the exposures may have been acquired during the rapid expansion phase (between phases of 0.9 and 1.0). The position and the width of spectral lines change rapidly during this phase and the measurements obtained from such spectra may not be reliable. Second, SSPP measures RVs by fitting a suitable spectral template to the entire observed (coadded) spectrum. This is not an optimal approach because the observed velocities measured from metallic and Balmer lines exhibit different behavior as a function of phase (Oke et al. 1962; Sesar 2012). Ideally, the systemic velocity should be determined for each line separately and then multiple estimates should be averaged out, as done in our Section 2.6. And third, SSPP uses
12 separate methods to measure $[\mathrm{Fe} / \mathrm{H}]$, none of which matches the one used in Section 2.7. The SSPP combines $[\mathrm{Fe} / \mathrm{H}]$ values from the various methods and provides an overall best value (FEHADOP) along with an uncertainty.

Out of the 27 RRab stars observed by us and by SDSS, only 10 stars have coadded SDSS spectra where the difference between the start of the first exposure and the end of the last exposure is less than an hour. The total exposure time of these coadded spectra is short enough $(<10 \%$ of the period) that the blurring of spectral lines is not significant. We corrected SSPP RVs for pulsation using corrections for the $\mathrm{H} \gamma$ line (these corrections gave the smallest scatter with respect to systemic velocities determined in Section 2.6). The average difference between our and SSPP velocities is $\sim-12 \mathrm{~km} \mathrm{~s}^{-1}$ and the rms scatter of differences is $\sim 20 \mathrm{~km} \mathrm{~s}^{-1}$. This scatter is fully consistent with uncertainties in estimated systemic velocities. Out of 10 RRab stars with good SDSS spectra, only 7 stars have $[\mathrm{Fe} / \mathrm{H}]$ measured by SSPP. The average difference between the $[\mathrm{Fe} / \mathrm{H}]$ measured in Section 2.7 and by SSPP is -0.36 dex and the rms scatter is 0.15 dex (i.e., SSSP metallicities are higher and the scatter is consistent with uncertainties). This value is similar to the -0.4 dex offset found by Drake et al. (2013a, see their Section 6.2.1).

In principle, we could average out our metallicity and velocity measurements with the ones determined by SSPP. However, adding SSPP measurements would not change any of the conclusions reported in following sections (e.g., the likelihood of membership) and may introduce systematic uncertainties that were not uncovered in the simple comparison presented in this section. Thus, we choose not to use SSPP data at this moment and plan to revisit this issue once properly measured SSPP velocities and metallicities become available for RRab stars (N. De Lee, in preparation).

\section{RESULTS}

Using data gathered and described in Section 2, we can now study heliocentric distances, velocities, and metallicities of RR Lyrae stars in the vicinity of the Orphan stream. To allow a direct 
comparison with Newberg et al. (2010) results, we adopt their definition and calculate the velocities in the Galactic standard of rest $\left(v_{\mathrm{gsr}}\right)$ as

$$
v_{\mathrm{gsr}}=v_{\text {helio }}+10.1 \cos b \cos l+224 \cos b \sin l+6.7 \sin b .
$$

Following Newberg et al. (2010), we have plotted the heliocentric distances, velocities, and metallicities as a function of galactic longitude $l$ in Figure 2. In subsequent sections, we use this figure to first select likely members of the Orphan stream, and then to examine various other properties of the stream, such as its metallicity and spatial extent.

\subsection{Orphan Stream Members}

In this section, we characterize the likelihood that an RRab star is a member of the Orphan stream. As we describe below, a star is assigned a high likelihood of being an Orphan stream member if

1. its galactic longitude is within $160^{\circ}<l<260^{\circ}$ and its sky position is within $4^{\circ}$ from the equator of the Orphan stream $\left(\left|B_{\text {Orphan }}\right|<4^{\circ}\right)$, and

2. its velocity in the Galactic standard of rest is $v_{\mathrm{gsr}}>$ $40 \mathrm{~km} \mathrm{~s}^{-1}$.

The heliocentric distance of a star is only a weak criterion and is used to increase (from low) or decrease (from high) the probability of three RRab stars to medium probability (see below). We emphasize that only RRab stars with high likelihood of membership are used when analyzing the metallicity of the stream (Section 3.2), its extent (Section 3.3), and when making comparisons with models (Section 3.4).

Looking at the top left panel of Figure 2, we can see that the best-fit orbits predict an increase in the heliocentric distance of the stream as the galactic longitude decreases, with the stream moving above $30 \mathrm{kpc}$ for $l<210^{\circ}$. At distances beyond $30 \mathrm{kpc}$, the number density of halo stars associated with the "smooth" stellar halo rapidly decreases (Sesar et al. 2010, 2011a; Deason et al. 2011), and as a result, halo substructures such as stellar streams are easier to discern (e.g., see Figure 11 of Sesar et al. 2010). Indeed, RR Lyrae stars in the vicinity of the Orphan stream and with $l<210^{\circ}$ seem to be distributed in an arc-like structure, which would be discernible even if we had no knowledge of the Orphan stream. The majority of stars in this region also have positive velocities in the Galactic standard of rest, as evident in the top right panel of Figure 2 $\left(v_{\mathrm{gsr}}>40 \mathrm{~km} \mathrm{~s}^{-1}\right)$, and these velocities agree with values observed by Newberg et al. (2010). Thus, we tag these stars as having a high probability of being Orphan stream members (see the last column in Tables 1 and 3).

For $l \lesssim 210^{\circ}$, and according to predicted orbits, the stream's heliocentric distance is expected to dip below $30 \mathrm{kpc}$. At these distances, the number density of RR Lyrae stars associated with the "smooth" halo is expected to be non-negligible, making the association of stars with the Orphan stream less straightforward. In this region, we rely on velocities to assign probabilities, and tag stars with $v_{\mathrm{gsr}}>40 \mathrm{~km} \mathrm{~s}^{-1}$ as having a high probability of being Orphan stream members.

For the most part, the stars can be easily tagged as having a high or low membership probability based on their position and velocity. However, there are three stars that were tagged as having a medium probability. RR5 is located at $l \sim 170^{\circ}$ and $D \sim 48 \mathrm{kpc}$, but has a very low velocity compared to other stars at this position $\left(v_{\mathrm{gsr}} \sim-2 \mathrm{~km} \mathrm{~s}^{-1}\right.$ versus $v_{\text {gsr }} \sim 100 \mathrm{~km} \mathrm{~s}^{-1}$ ). Since RR Lyrae stars are standard candles, the heliocentric distance is considered reliable; this star is likely located at $D>40 \mathrm{kpc}$ even if we assume its metallicity to be $[\mathrm{Fe} / \mathrm{H}]=-1.0 \mathrm{dex}$. In the case of this star, the measured velocity is more likely to be incorrect than the distance, and additional velocity observations may resolve this discrepancy. If the currently measured velocity is wrong, then we believe this star is a likely member of the Orphan stream.

Unlike RR5, RR20 and RR22 have velocities consistent with those of Orphan stream stars at $l \sim 200^{\circ}\left(v_{\mathrm{gsr}} \sim 100 \mathrm{~km} \mathrm{~s}^{-1}\right)$, but are much closer than the predicted orbit of the stream $(23 \mathrm{kpc}$ versus $35 \mathrm{kpc}$ for the stream). In addition, these stars have a metallicity of $[\mathrm{Fe} / \mathrm{H}] \sim-1.6$ dex, making them rather metalrich for the Orphan stream. Unless the Orphan stream has a much greater line-of-sight breadth than previously thought, we consider these stars as less likely members of the Orphan stream. As for CSS RRab stars without spectroscopic observations, shown as open squares in Figure 1 and the top left panel of Figure 2, we cannot estimate likelihood of their membership without spectroscopic data (i.e., RVs), even for stars that are seemingly near the predicted orbit of the Orphan stream. Stars without spectroscopic observations are not used in our analysis and their follow up is encouraged.

We also considered using proper motions as additional criteria for assigning membership probabilities. Unfortunately, the range of proper motions for likely and unlikely members was too small relative to the uncertainty in measurements to make

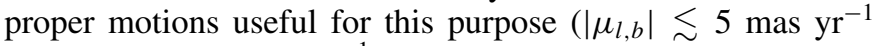

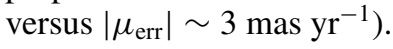

\subsection{Metallicity of the Orphan Stream}

Since the metallicity was not used as a criterion while assigning membership probabilities, it would be interesting to examine the distribution of metallicities for stars that are likely or unlikely to be associated with the Orphan stream.

The bottom left panel of Figure 2 shows the metallicity of RR Lyrae stars along the Orphan stream (i.e., in the direction of galactic longitude). Here we have assumed that the uncertainty in metallicity is 0.15 dex for all stars. We consider this error to be slightly overestimated. As stated in Section 2.7, a comparison of measured and literature values of metallicities of four bright RR Lyrae stars indicates that the systematic uncertainty in metallicity is about 0.07 dex. Furthermore, one of the stars in our sample (RR6) was observed on two different nights, ${ }^{15}$ and the measured metallicities were -2.45 dex and -2.29 dex. The rms scatter of these two measurements is 0.11 dex, even though the S/N per $\AA$ of both observations was only 12 .

As the bottom left panel shows, likely members of the Orphan stream span a wide range of metallicities, from -1.5 dex to -2.7 dex. Stars that are unlikely to be associated with the Orphan stream also span a wide range, from -1.3 dex to -2.3 dex. The two samples overlap significantly in metallicity, making the metallicity a weak criterion for identifying members of the Orphan stream. The velocity in the Galactic standard of rest is a more powerful criterion, as evident from the bottom right panel of Figure 2.

The metallicity versus position panel does show an interesting trend. While the stars not associated with the Orphan stream seem to span a wide range of metallicities regardless of their

\footnotetext{
15 Its velocity and the metallicity listed in Table 3 are average values.
} 
position, stars associated with the stream seem to be increasingly more metal-rich in the eastward direction. To verify this, we have binned likely members into two groups and calculated the mean metallicity and the standard error of the mean for each group. We find that the stars located near $l \sim 210^{\circ}$ are 0.3 dex more metal-rich than the stars near $l \sim 180^{\circ}$ (the standard error of the mean is $\sim 0.06 \mathrm{dex}$ ).

The statistical dependence between the galactic longitude of a star and its metallicity can be tested using the Kendall's $\tau_{B}$ coefficient (e.g., see Lupton 1993). This test is non-parametric, as it does not rely on any assumptions on the distributions of $X$ or $Y$ or the distribution of $(X, Y)$. Values of $\tau_{B}$ range from -1 (perfect inversion) to 1 (perfect agreement). A value of zero indicates the absence of association. Using 27 highly likely members with $l<240^{\circ}$ (to minimize possible contamination by stars associated with the smooth halo spheroid, see below) we obtained $\tau_{B}=0.416 \pm 0.002$, indicating statistically significant correlation between galactic longitude of an Orphan stream RRab star and its metallicity. The correlation between $[\mathrm{Fe} / \mathrm{H}]$ and $\Lambda_{\text {Orphan }}$ (the position along the Orphan stream) is slightly stronger and is $\tau_{B}=0.433 \pm 0.002$.

To estimate the probability that the observed correlation is due to random chance, we generate 10,000 samples of 27 stars and count the number of samples where $\tau_{B}>0.416$ and the uncertainty in $\tau_{B}<0.002$. The samples are generated by drawing metallicities from a 0.3 dex wide Gaussian centered on -2.1 dex (i.e., the metallicity distribution of highly likely Orphan stream members), while keeping the galactic longitude of stars unchanged. We find that the probability of seeing the observed correlation due to random chance is $\lesssim 10^{-3}$. The same result is obtained if instead of drawing metallicities, we randomly exchange positions of stars used in this analysis.

It is not impossible that the observed $[\mathrm{Fe} / \mathrm{H}]$ versus $l$ correlation is due to unidentified contamination by RRab stars actually associated with the smooth halo spheroid. Since the metallicity of halo stars is centered on $[\mathrm{Fe} / \mathrm{H}]=-1.5$ dex (Ivezić et al. 2007), unidentified contamination could make the Orphan stream seem more metal-rich as it gets closer to the Galactic plane, that is, as the galactic longitude increases. Because the unidentified contamination and a metallicity gradient could both make the Orphan stream seem more metal-rich, we cannot use the $[\mathrm{Fe} / \mathrm{H}]$ versus $l$ distribution (i.e., the bottom left panel of Figure 2) to make any estimates of the contamination. Velocities, however, should not be affected by the metallicity gradient, which is why they can be used to make statements on the level of contamination.

Based on the velocity distribution of stars in the top right panel of Figure 2, we do not think the metallicity gradient is caused by unidentified contamination by halo stars. If the contamination was significant, then there would be more RRab stars with $v_{\text {gsr }} \sim 0 \mathrm{~km} \mathrm{~s}^{-1}$, since the RV distribution of halo stars is a $\sim 100 \mathrm{~km} \mathrm{~s}^{-1}$ wide Gaussian centered on zero (Bond et al. 2010). Instead, the distribution of velocities in the top right panel of Figure 2 is clearly bimodal, with stars having velocities at the extreme of the halo velocity distribution. The only exception may be stars at $l>240^{\circ}$, which is why we excluded them when studying the correlation between the galactic longitude of an Orphan stream RRab star and its metallicity.

In Section 2.7 we stated that the measured [Ca II] pseudoequivalent widths, $\mathrm{W}(\mathrm{K})$, were corrected for interstellar [Ca II] absorption using the Beers (1990) model. At large distances from the Galactic plane, this model is equal to $W\left(K_{\text {ism }}\right)=$ $0.192 / \sin (|b|)$, where $b$ is the galactic latitude. Since the two groups of stars are at different galactic latitudes $\left(48^{\circ}\right.$ and $\left.53^{\circ}\right)$, it is not impossible that the observed gradient is caused by this correction. To verify whether this is the case, we estimated the metallicities without correcting the $W(K)$ for interstellar [Ca II] absorption, and compared them to values listed in Table 3. The metallicities that were not corrected for interstellar [Ca II] absorption were higher by $0.1 \mathrm{dex}$, but that offset was the same for both groups. Thus, we conclude that the observed gradient is not due to the correction for interstellar [Ca II] absorption.

\subsection{Does the Orphan Stream End at $55 \mathrm{kpc}$ ?}

One of the goals of this work was to trace the Orphan stream beyond the $46 \mathrm{kpc}$ limit reached by Newberg et al. (2010). As the top left panel of Figure 2 shows, we have achieved that goal. The question that we now ask is does the Orphan stream extend further into the halo or not?

The question of how far the Orphan stream reaches into the halo is an important one. As the curved lines in the top left panel of Figure 2 show, the heliocentric distance of the Orphan stream constrains the halo potential at large distances. If there are Orphan stream RRab stars beyond $55 \mathrm{kpc}$, they should be followed-up spectroscopically because their properties could be used to even better constrain the halo potential and the orbit of the stream. Alternatively, a lack of Orphan stream RRab stars beyond $55 \mathrm{kpc}$ may indicate that we have detected the end of the leading arm of the stream, ${ }^{16}$ and such a finding would be important for future theoretical and observational studies of the stream.

To make sure we did not miss RRab stars due to an overly restrictive spatial cut, we defined a region on the sky (dotted box in Figure 1), and carefully searched the PTF data set in this region for distant RR Lyrae stars that may have been missed. The result of this search: no additional RRab stars were identified.

To estimate the incompleteness of our search, we ran our selection algorithm on a sample of 100 simulated RR Lyrae light curves. The light curves were created by sampling 26 data points from a template RRab light curve with a maximum brightness of $m_{0}=18.5 \mathrm{mag}$, amplitude of $1.0 \mathrm{mag}$, and a period of 0.53 days. These parameters correspond to best-fit parameters of the most distant RRab star observed in this work (RR6; see Table 1). The epoch of maximum light was randomly selected for each simulated light curve, and the light curves were sampled at Julian dates of PTF observations in the region of interest. To make the light curves realistic, we added Gaussian noise to sampled magnitudes using a model that describes the mean photometric uncertainty in the PTF $R$-band as a function of magnitude.

We found that an RRab star at $\sim 50 \mathrm{kpc}$ and with an amplitude of 1 mag may not be recovered $13 \%$ of the time (i.e., the recovery fraction is 87\%). A star at the same distance, but with a lower amplitude of 0.5 mag may be missed $34 \%$ of the time. At $60 \mathrm{kpc}$, the incompleteness is $20 \%$ and $46 \%$ for RRab stars with amplitudes of 1 mag and 0.5 mag, respectively. Thus, it is possible that some distant RRab stars were not detected due to insufficient $\mathrm{S} / \mathrm{N}$ in PTF data. We intend to obtain additional observations of this region with PTF to fully explore this possibility.

The boxed region, shown in Figure 1, does contain a single CSS RRab star (RR4), which we observed spectroscopically.

\footnotetext{
16 Based on the surface density profile and the velocities of Orphan stream stars, Newberg et al. (2010) have concluded that the part stretching from $l=250^{\circ}$ to $l=170^{\circ}$ is the leading tidal arm.
} 
Table 4

Orphan Stream Models of Newberg et al. (2010)

\begin{tabular}{ccccccccccc}
\hline \hline$N$ & $\begin{array}{c}M_{\text {Bulge }} \\
\left(10^{10} M_{\odot}\right)\end{array}$ & $\begin{array}{c}\text { Disk } \\
\text { Type }\end{array}$ & $\begin{array}{c}M_{\text {disk }} \\
\left(10^{10} M_{\odot}\right)\end{array}$ & $\begin{array}{c}\text { Halo } \\
\text { Type }\end{array}$ & $\begin{array}{c}M_{\text {halo }} \\
\left(10^{10} M_{\odot}\right)\end{array}$ & $\begin{array}{c}d / r_{s} \\
(\mathrm{kpc})\end{array}$ & $\begin{array}{c}v_{\text {halo }} \\
\left(\mathrm{km} \mathrm{s}^{-1}\right)\end{array}$ & $\begin{array}{c}M_{60} \mathrm{a}^{10} \\
\left(10^{10} M_{\odot}\right)\end{array}$ & $\chi^{2 \mathrm{~b}}$ & Fits $V_{c}^{\mathrm{c}}$ \\
\hline 1 & 1.5 & Exp & 5 & NFW & 33 & 22.25 & 155 & 40 & 2.8 & No \\
2 & 1.5 & Exp & 5 & NFW & 20 & 22.25 & $120 \pm 7$ & 24 & 3.5 & No \\
3 & 1.5 & Exp & 5 & Log & 17.6 & 12 & $81 \pm 12$ & 26.5 & 3.5 & No \\
4 & 3.4 & M-N & 10 & Log & 35 & 12 & 114 & 47 & 4.3 & Yes \\
5 & 3.4 & M-N & 10 & Log & 14 & 12 & $73 \pm 24$ & 26.4 & 2.3 & Yes \\
6 & 3.4 & M-N & 10 & NFW & 33 & 22.25 & 155 & 43.5 & 10.0 & Yes \\
7 & 3.4 & M-N & 10 & NFW & 16 & 22.25 & $109 \pm 31$ & 28.4 & 2.3 & Yes \\
\hline
\end{tabular}

Notes. A portion of this table has been copied from Table 3 of Newberg et al. (2010).

a Mass due to sum of bulge, disk, and halo components out to $R=60 \mathrm{kpc}$.

${ }^{b}$ Reduced $\chi^{2}$ of the orbit fit to distances of likely Orphan stream RRab stars located beyond $33 \mathrm{kpc}$.

c Circular velocity of $V_{c} \sim 220 \mathrm{~km} \mathrm{~s}^{-1}$ (Koposov et al. 2010; Bovy et al. 2012) near the Sun's radius (see

Figures 13 and 15 of Newberg et al. 2010).

However, we did not additionally search CSS in this region for distant RRab stars that may have been missed by Drake et al. (2013a) because CSS is shallower than PTF. As shown by Drake et al. (2013a), their catalog of RR Lyrae stars selected from CSS is about $60 \%$ complete up to $V \sim 17.5 \mathrm{mag}$ ( $\sim 25 \mathrm{kpc})$, after which completeness decreases (see their Figure 13). The Mount Lemmon Survey (MLS), which is deeper than CSS by 2 mag (Larson 2007) and which was used by Drake et al. (2013b) to discover a potential new stream in the outer halo, does not cover the far end of the Orphan stream (see Figure 14 of Drake et al. 2013b).

Even if there are no RR Lyrae stars associated with the Orphan stream at heliocentric distances greater than $\sim 55 \mathrm{kpc}$, this does not necessarily mean that the stream ends at $\sim 55 \mathrm{kpc}$. As the bottom left panel of Figure 2 shows, the average metallicity of RRab stars in the Orphan stream seems to be decreasing with decreasing galactic longitude, or equivalently, with increasing heliocentric distance. At some point, the metallicity of Orphan stream stars may be low enough that the horizontal branch of such metal-poor stars is completely devoid of RR Lyrae stars, and is instead populated by BHB stars. BHB stars do not pulsate and we cannot detect them using our data. Thus, it is possible that the Orphan stream extends further into the halo, but we are simply unable to trace it there using RR Lyrae stars.

\subsection{Comparison with Best-fit Orbits of Newberg et al. (2010)}

The positions and velocities of RR Lyrae stars associated with the Orphan stream allow us to make comparisons with orbits obtained by Newberg et al. (2010). To fit these orbits, Newberg et al. (2010) used seven models for the potential: three models published by previous authors (models 1, 4, and 6 ), and four models in which the halo mass was varied to best match their Orphan stream data (logarithmic and NavarroFrenk-White (NFW) halos were compared, as well as a lowmass exponential disk versus a high-mass Miyamoto-Nagai (M-N) disk; see Section 10.1 of Newberg et al. 2010). Their models are summarized in Table 4.

A comparison of the heliocentric distances of RRab stars and best-fit orbits is shown in the top left panel of Figure 2. The reduced $\chi^{2}$ values listed in Table 4 quantify how well the orbits fit distances of likely RRab members located beyond $33 \mathrm{kpc}$ from the Sun.

The poorest fit is provided by the orbit based on model 6 (reduced $\chi^{2}=10$ ). Model 6 is very similar to model 1 , which is based on parameters of Xue et al. (2008), but instead of the
$M_{\text {disk }}=5 \times 10^{10} M_{\odot}$ exponential disk used in model 1 , it uses a more massive $M_{\text {disk }}=10 \times 10^{10} M_{\odot}$ M-N disk. The orbit based on model 6 clearly does not fit distances of RRab stars located beyond $46 \mathrm{kpc}$. Thus, we conclude that the potential of the Milky Way is likely not similar to the one described by model 6.

The orbits based on models 1,2 , and 3 provide reasonable fits to distances of remote Orphan stream RRab stars (reduced $\chi^{2} \sim$ 3 ). However, as shown in Figure 13 of Newberg et al. (2010), these models predict circular velocities near the Sun's radius that are too low $\left(V_{c}<190 \mathrm{~km} \mathrm{~s}^{-1}\right)$ compared to measurements obtained by Koposov et al. $\left(2010, V_{c}=221 \pm 18 \mathrm{~km} \mathrm{~s}^{-1}\right)$ and Bovy et al. $\left(2012, V_{c}=218 \pm 6 \mathrm{~km} \mathrm{~s}^{-1}\right)$.

On the other hand, models 4, 5, and 7 fit the local circular velocity measurement of Koposov et al. (2010) (see Figure 15 of Newberg et al. 2010). The distances of remote Orphan stream RRab stars are also best fit by models 5 and 7 (reduced $\chi^{2}=2.3$ ). Based on this comparison, we conclude that models 5 and 7 represent the best description of the Milky Way potential within $60 \mathrm{kpc}$ (we have a slight preference for model 7 because it better fits the Koposov et al. (2010) measurement).

Models 5 and 7 have different halos (logarithmic versus NFW), but with the data at hand and based on best-fit orbits of Newberg et al. (2010), we are not able to distinguish between these two types of halos. The important characteristic of these two models is the total mass within $60 \mathrm{kpc}$, which is $M_{60} \sim 2.7 \times 10^{11} M_{\odot}$, or about $60 \%$ of the mass within $60 \mathrm{kpc}$ found by previous studies (Law et al. 2005; Xue et al. 2008; Deason et al. 2012).

Compared to heliocentric distances, the velocities of RRab stars (top right panel of Figure 2) are not as useful for discriminating models. Near $l=250^{\circ}$, the median velocity of $\mathrm{RRab}$ stars is about $30 \mathrm{~km} \mathrm{~s}^{-1}$ lower than the predicted velocity (75 $\mathrm{km} \mathrm{s}^{-1}$ versus $105 \mathrm{~km} \mathrm{~s}^{-1}$ ). Although this is interesting, note that the sample of Orphan stream RRab stars is quite sparse near this longitude, so it is difficult to make any strong conclusions.

\section{DISCUSSION AND CONCLUSIONS}

In this work, we used RR Lyrae stars to trace the Orphan stream in the northern Galactic hemisphere, with a focus on tracing it beyond the $46 \mathrm{kpc}$ limit reached by Newberg et al. (2010). The main goal of this work was to support future studies of the Galactic potential by providing a clean sample of Orphan stream RR Lyrae stars with precise distances and RVs (better 
than $5 \%$ and $15 \mathrm{~km} \mathrm{~s}^{-1}$, respectively). The secondary goal of this work was to use the kinematics and positions of RR Lyrae stars associated with the Orphan stream to see whether new measurements exclude any of the models previously considered by Newberg et al.

In total, we have observed $50 a b$-type RR Lyrae stars in the vicinity of the Orphan stellar stream and measured their distances, velocities, and metallicities. Using these data we have classified about 30 RRab stars as being likely members of the Orphan stream, the most distant of which is located at about $55 \mathrm{kpc}$, or about $9 \mathrm{kpc}$ beyond the limit reached by Newberg et al. (2010).

We find that RRab stars in the Orphan stream have a wide range of metallicities, from -1.5 dex to -2.7 dex. The average metallicity of RRab stars in the Orphan stream is -2.1 dex (standard deviation is $0.3 \mathrm{dex}$ ), and is identical to the metallicity of BHB stars measured by Newberg et al. (2010). The most distant parts of the stream $(40-50 \mathrm{kpc}$ from the Sun) are about 0.3 dex more metal-poor than the closer parts (within $\sim 30 \mathrm{kpc}$ ), suggesting a possible metallicity gradient along the stream's length. According to the Kendall's $\tau$ test, the correlation between the galactic longitude of an Orphan stream RRab star and its metallicity seems to be statistically significant $\left(\tau_{B}=0.416 \pm 0.002\right)$. Using Monte Carlo simulations, we have determined that the probability of seeing the observed correlation due to random chance is $\lesssim 10^{-3}$.

If real, this gradient may reconcile the average metallicity found by Casey et al. (2013) ( -1.6 dex at Orphan longitude $\Lambda_{\text {Orphan }} \sim 20^{\circ}$ ) with the metallicities measured in this work and by Newberg et al. (2010) $\left(-2.1\right.$ dex for $\Lambda_{\text {Orphan }}<-10^{\circ}$ ). Since the only other stellar stream with a known metallicity gradient is the Sagittarius stream (Chou et al. 2007), adding the Orphan stream to this list may provide additional constraints on models of the chemical evolution of dwarf spheroidal galaxies, the evolution of dwarf galaxies, and the process of tidal stripping and disruption.

The presence of a metallicity gradient could be tested by observing K giants associated with the Orphan stream. As shown by Casey et al. (2013), the most difficult part of such a study is the identification of likely $\mathrm{K}$ giants in the stream. One way to identify likely candidates would be to use a gravity-sensitive filter (such as DDO51) in combination with a wide-field imager to separate dwarfs and giants (e.g., as done by Morrison et al. 2001). Once candidate giants are identified, their classification could be further improved by comparing their position in a color-magnitude diagram to an isochrone placed at the distance of RRab stars in the Orphan stream.

Since the heliocentric distance of the Orphan stream can constrain the potential of the Milky Way (see below), we attempted to trace the stream as far into the halo as possible. Although we successfully mapped the stream to about $55 \mathrm{kpc}$ from the Sun (about $9 \mathrm{kpc}$ further than Newberg et al. 2010), we did not identify more distant RRab stars that could plausibly be Orphan stream members. One explanation for this may be the incompleteness of our selection, which ranges from $20 \%$ to $46 \%$ at $60 \mathrm{kpc}$ and for stars with amplitudes from $1 \mathrm{mag}$ to $0.5 \mathrm{mag}$, respectively. We intend to obtain additional observations of this region with PTF to fully explore this possibility. The metallicity gradient could also explain the lack of distant RRab stars in the Orphan stream. At some point, the metallicity of Orphan stream stars may be low enough that the horizontal branch of such metal-poor stars is completely devoid of RR Lyrae stars, and is instead populated by BHB stars. And finally, the lack of
RR Lyrae stars may indicate that we have detected the end of the leading arm of the Orphan stream. Detections and follow-up observations of $\mathrm{K}$ giants may provide additional support for this hypothesis.

We have compared the distances of Orphan stream RRab stars with several best-fit orbits obtained by Newberg et al. (2010), and found that the model 6 of Newberg et al. (2010) cannot explain the heliocentric distances of the most remote Orphan stream RRab stars. Following similar arguments as Newberg et al. (2010), we find that the distances of RRab stars prefer potentials where the total mass of the Galaxy within $60 \mathrm{kpc}$ is $M_{60} \sim 2.7 \times 10^{11} M_{\odot}$, or about $60 \%$ of that found by Law et al. (2005), Xue et al. (2008), and Deason et al. (2012). A similar conclusion was also reached by Newberg et al. (2010).

There is an issue one should be aware of when considering the total mass of the Galaxy deduced here. In Section 1, we said that the misalignment between the stream and the orbit may be small enough that fitting an orbit directly to the stream has no significant consequences for the inferred shape of the Galactic potential (Sanders \& Binney 2013a). However, there may be an additional misalignment between the stream and the orbit due to the spread in actions of the stream, caused by a substantial mass and size of the progenitor. This misalignment may render orbit-fitting, and the potentials inferred from it, less reliable. In case of the Orphan stream, there seems to be some evidence of a spread in actions, since the velocity dispersion of BHB stars in the Orphan stream $\left(\sim 10 \mathrm{~km} \mathrm{~s}^{-1}\right.$; Newberg et al. 2010) cannot be fully accounted for by the uncertainty in their RVs $\left(\sim 5 \mathrm{~km} \mathrm{~s}^{-1}\right)$. Fortunately, alternatives to orbit-fitting already exist (e.g., Sanders \& Binney 2013b), and may yield more reliable results when applied to the Orphan stream.

A better differentiation between various models of the Galactic potential may be achieved by improving the precision of RRab distances. This can be accomplished by observing RRab stars in the near-infrared, where the slope of their period-luminosity relation (i.e., the Leavitt law) is steeper and width/scatter in the relation is smaller (Madore \& Freedman 2012). The IRAC camera on board the Spitzer Space Telescope would be the perfect instrument for this purpose, and we intend to propose such a study in the near future.

B.S. and J.G.C. thank NSF grant AST-0908139 to J.G.C. for partial support, as do S.R.K. (to NSF grant AST-1009987), and C.J.G. (for a NASA grant). E.O.O. is incumbent of the Arye Dissentshik career development chair and is grateful to support by a grant from the Israeli Ministry of Science and the I-CORE Program of the Planning and Budgeting Committee and The Israel Science Foundation (grant No 1829/12). We thank the staff at the Palomar Hale telescope for help and support with observations. We thank the anonymous referee for the careful reading of our manuscript and the valuable comments.

This article is based on observations obtained with the Samuel Oschin Telescope as part of the Palomar Transient Factory project, a scientific collaboration between the California Institute of Technology, Columbia University, Las Cumbres Observatory, the Lawrence Berkeley National Laboratory, the National Energy Research Scientific Computing Center, the University of Oxford, and the Weizmann Institute of Science. It is also partially based on observations obtained as part of the Intermediate Palomar Transient Factory project, a scientific collaboration among the California Institute of Technology, Los Alamos National Laboratory, the University of Wisconsin, Millwakee, the Oskar Klein Center, the Weizmann Institute of 
Science, the TANGO Program of the University System of Taiwan, the Kavli Institute for the Physics and Mathematics of the Universe, and the Inter-University Centre for Astronomy and Astrophysics.

\section{REFERENCES}

Beers, T. C. 1990 , AJ, 99, 323

Bell, E. F., Xue, X. X., Rix, H.-W., Ruhland, C., \& Hogg, D. W. 2010, AJ, 140,1850

Belokurov, V., Evans, N. W., Irwin, M. J., et al. 2007a, ApJ, 658, 337

Belokurov, V., Zucker, D. B., Evans, N. W., et al. 2007b, ApJ, 654, 897

Bhalerao, V. 2012, PhD thesis, Caltech

Bickerton, S., Badenes, C., Hettinger, T., Beers, T., \& Huang, S. 2012, in IAU Symp. 285, New Horizons in Time-Domain Astronomy, ed. E. Griffin, R. Hanisch, \& R. Seaman (Cambridge: Cambridge Univ. Press), 289

Bond, N. A., Ivezić, Ž., Sesar, B., et al. 2010, ApJ, 716, 1

Bovy, J., Allende Prieto, C., Beers, T. C., et al. 2012, ApJ, 759, 131

Cacciari, C., \& Clementini, G. 2003, in Stellar Candles for the Extragalactic Distance Scale, ed. D. Alloin \& W. Gieren (Lecture Notes in Physics, Vol. 635; Berlin: Springer), 105

Casey, A. R., Da Costa, G., Keller, S. C., \& Maunder, E. 2013, ApJ, 764, 39

Chaboyer, B. 1999, in Post-Hipparcos Cosmic Candles, ed. A. Heck \& F. Caputo (Astrophysics and Space Science Library, Vol. 237; Dordrecht: Kluwer), 111

Chou, M.-Y., Majewski, S. R., Cunha, K., et al. 2007, ApJ, 670, 346

Dall'Ora, M., Kinemuchi, K., Ripepi, V., et al. 2012, ApJ, 752, 42

Deason, A. J., Belokurov, V., \& Evans, N. W. 2011, MNRAS, 416, 2903

Deason, A. J., Belokurov, V., Evans, N. W., \& An, J. 2012, MNRAS, 424, L44

Drake, A. J., Catelan, M., Djorgovski, S. G., et al. 2013a, ApJ, 763, 32

Drake, A. J., Catelan, M., Djorgovski, S. G., et al. 2013b, ApJ, 765, 154

Drake, A. J., Djorgovski, S. G., Mahabal, A., et al. 2009, ApJ, 696, 870

Grillmair, C. J. 2006, ApJL, 645, L37

Grillmair, C. J., \& Dionatos, O. 2006, ApJL, 643, L17

Horne, K. 1986, PASP, 98, 609

Ivezić, Ž., Smith, J. A., Miknaitis, G., et al. 2007, AJ, 134, 973

Koposov, S. E., Rix, H.-W., \& Hogg, D. W. 2010, ApJ, 712, 260

Larson, S. 2007, in IAU Symp. 236, Near Earth Objects, our Celestial Neighbors: Opportunity and Risk, ed. G. B. Valsecchi, D. Vokrouhlický, \& A. Milani (Cambridge: Cambridge Univ. Press), 323

Law, D. R., Johnston, K. V., \& Majewski, S. R. 2005, ApJ, 619, 807

Law, D. R., \& Majewski, S. R. 2010, ApJ, 714, 229
Law, N. M., Kulkarni, S. R., Dekany, R. G., et al. 2009, PASP, 121, 1395

Layden, A. C. 1994, AJ, 108, 1016

Lee, Y. S., Beers, T. C., Allende Prieto, C., et al. 2011, AJ, 141, 90

Lee, Y. S., Beers, T. C., Sivarani, T., et al. 2008, AJ, 136, 2022

Lupton, R. 1993, Statistics in Theory and Practice (Princeton, NJ: Princeton Univ. Press)

Madore, B. F., \& Freedman, W. L. 2012, ApJ, 744, 132

Majewski, S. R., Skrutskie, M. F., Weinberg, M. D., \& Ostheimer, J. C. 2003, ApJ, 599, 1082

Martin, N. F., Ibata, R. A., Chapman, S. C., Irwin, M., \& Lewis, G. F. 2007, MNRAS, 380, 281

Martinez, G. D., Minor, Q. E., Bullock, J., et al. 2011, ApJ, 738, 55

Morrison, H. L., Olszewski, E. W., Mateo, M., et al. 2001, AJ, 121, 283

Munari, U., Sordo, R., Castelli, F., \& Zwitter, T. 2005, A\&A, 442, 1127

Munn, J. A., Monet, D. G., Levine, S. E., et al. 2004, AJ, 127, 3034

Newberg, H. J., Willett, B. A., Yanny, B., \& Xu, Y. 2010, ApJ, 711, 32

Ofek, E. O., Frail, D. A., Breslauer, B., et al. 2011, ApJ, 740, 65

Ofek, E. O., Laher, R., Law, N., et al. 2012a, PASP, 124, 62

Ofek, E. O., Laher, R., Surace, J., et al. 2012b, PASP, 124, 854

Oke, J. B., Giver, L. P., \& Searle, L. 1962, ApJ, 136, 393

Oke, J. B., \& Gunn, J. E. 1982, PASP, 94, 586

Rau, A., Kulkarni, S. R., Law, N. M., et al. 2009, PASP, 121, 1334

Sanders, J. L., \& Binney, J. 2013a, MNRAS, 433, 1813

Sanders, J. L., \& Binney, J. 2013b, MNRAS, 433, 1826

Schlegel, D. J., Finkbeiner, D. P., \& Davis, M. 1998, ApJ, 500, 525

Sesar, B. 2012, AJ, 144, 114

Sesar, B., Cohen, J. G., Levitan, D., et al. 2012, ApJ, 755, 134

Sesar, B., Ivezić, Ž., Grammer, S. H., et al. 2010, ApJ, 708, 717

Sesar, B., Ivezić, Ž., Stuart, J. S., et al. 2013, AJ, 146, 21

Sesar, B., Jurić, M., \& Ivezić, Ž. 2011a, ApJ, 731, 4

Sesar, B., Stuart, J. S., Ivezić, Ž., et al. 2011b, AJ, 142, 190

Sirko, E., Goodman, J., Knapp, G. R., et al. 2004, AJ, 127, 899

Stokes, G. H., Evans, J. B., Viggh, H. E. M., Shelly, F. C., \& Pearce, E. C. 2000, Icar, 148, 21

Vera-Ciro, C., \& Helmi, A. 2013, ApJL, 773, L4

Vickers, J. J., Grebel, E. K., \& Huxor, A. P. 2012, AJ, 143, 86

Vivas, A. K., \& Zinn, R. 2006, AJ, 132, 714

Xue, X. X., Rix, H. W., Zhao, G., et al. 2008, ApJ, 684, 1143

Yanny, B., Newberg, H. J., Johnson, J. A., et al. 2009a, ApJ, 700, 1282

Yanny, B., Rockosi, C., Newberg, H. J., et al. 2009b, AJ, 137, 4377

York, D. G., Adelman, J., Anderson, J. E., Jr., et al. 2000, AJ, 120, 1579

Zinn, R., \& West, M. J. 1984, ApJS, 55, 45

Zucker, D. B., Belokurov, V., Evans, N. W., et al. 2006, ApJL, 650, L41 